\title{
EVALUACIÓN POBLACIONAL DEL STOCK EXPLOTABLE DEL COMPLEJO OPISTHONEMA (PISCES: CLUPEIDAE) EN EL GOLFO DE NICOYA, COSTA RICA
}

\author{
Luis A. Vega Corrales ${ }^{1,2}$
}

\begin{abstract}
RESUMEN
El género Opisthonema sustenta la pesquería de sardina en Costa Rica. Esta investigación se orientó hacia la evaluación poblacional del stock explotable de esta pesquería en el Golfo de Nicoya. Las muestras mensuales fueron recolectadas desde junio del 2007 hasta mayo del 2008. Se determinó el patrón de reproducción de $O$. libertate y se estimaron los parámetros de crecimiento de las curvas en longitud del modelo de von Bertalanffy para $O$. libertate y $O$. medirastre. Con base en el índice gonadosomático, O. libertate desova intensamente durante la época más calurosa del año, entre febrero y mayo. La talla de primera madurez $\left(\mathrm{TPM}_{50 \%}\right)$ fue de $18.68 \mathrm{~cm}$ de LT. La ecuación de crecimiento de von Bertalanffy estimada para $O$. libertate fue: $\mathrm{Lt}=25.9\left[1-\exp ^{-1.03(t+0.13)}\right]$ y para $O$. medirastre: $\mathrm{Lt}=32.0\left[1-\exp ^{-1.10(t+0.15)}\right]$. El reclutamiento máximo de la pesquería de $O$. libertate ocurre entre septiembre y noviembre. Esta especie muestra un índice de explotación (E) de 0.65 y O. medirastre de 0.55 . Se nota una mejoría en el E comparado con estimaciones anteriores. Se recomienda como medida de manejo para esta pesquería ajustar la época de veda y no incrementar el esfuerzo pesquero.
\end{abstract}

Palabras claves: Sardina, Opisthonema, evaluación poblacional, Golfo de Nicoya, Costa Rica.

\begin{abstract}
The Opisthonema genus supports the sardine fishery in Costa Rica. This research was directed to population assessment of the exploitable stock of this fishery in the Nicoya Gulf. Monthly samples were collected from June 2007 to May 2008. The reproductive pattern of $O$. libertate was determined and growth parameters of curves in the length of $O$. libertate and $O$. medirastre were estimated using the von Bertalanffy model. Based on the gonadosomatic index, $O$. libertate spawn intensively during the hottest time of the year, between February and May. The size at first maturity $\left(\mathrm{SFM}_{50 \%}\right)$ was $18.68 \mathrm{~cm}$ T.L. The growth equation of von Bertalanffy was estimated for $O$. libertate: $\mathrm{Tl}=25.9\left[1-\exp ^{-1.03\left(+t^{+0.13}\right)}\right]$ and O. medirastre: $\mathrm{Tl}=32.0$ $\left[1-\exp ^{-1.10(t+0.15)}\right]$. The maximum recruitment to the fishery of $O$. libertate occurs between September and November. This species shows an exploitation index (E) of 0.65 and O. medirastre has an E value of 0.55. There is an improvement in the $\mathrm{E}$ value, as compared to previous values. It is recommended as a management measure to adjust the closing season and to avoid any increment of the fishing effort.
\end{abstract}

Keywords: Sardine, Opisthonema, population assessment, Nicoya Gulf, Costa Rica.

\section{INTRODUCCIÓN}

Las tres especies del género Opisthonema (O. libertate, O. medirastre y O. bulleri) sustentan la pesquería de sardina en Costa Rica. A este grupo de especies se les puede definir como complejo Opisthonema, debido a que ocupan las mismas áreas de pesca, son fenotípicamente parecidas, tienen el mismo interés o valor comercial y son capturadas con las mismas artes de pesca (Csirke, 1980; Stevenson y Carranza, 1981).

Estudios previos sobre este género en el país se han basado en la taxonomía

1 PROCMAR-UNA, Programa de Maestría de Ciencias Marinas y Costeras, Universidad Nacional, Costa Rica.

2 EBM-UNA, Estación de Biología Marina, Universidad Nacional, Costa Rica. lvega@una.ac.cr 
(Bussing y López, 1993; Berry y Barrett, 1963), la pesquería (Stevenson y Carranza, 1981), los parámetros poblacionales (Rodríguez et al. 1999; Soto, 1999; Soto y Rodríguez, 1999), la reproducción (Rodríguez et al. 1986, 1989, 1999; Soto, 1999) y el crecimiento (Víquez et al. 1990, 1992).

Investigaciones pesqueras han demostrado que los parámetros poblacionales de una especie cambian en relación con el tiempo, y dependiendo del grado de explotación de la población, su talla de primera madurez varía como estrategia reproductiva para alcanzar un nuevo punto de equilibrio biológico (Gulland, 1971; Csirke, 1980). De esta manera, considerar a la población íctica en estudio como un ente que cambia de tamaño, estructura y composición, justifica su monitoreo en el tiempo y la identificación de las circunstancias exógenas que la afectan, como por ejemplo, la mortalidad por pesca, que es la principal causa en la reducción de poblaciones de peces y que, finalmente, es la que se puede detectar y controlar (Sparre y Venema, 1995).

El objetivo de esta investigación fue determinar la distribución por especie, el período de desove, la talla de primera madurez y la dinámica poblacional del stock explotable del complejo Opisthonema en el Golfo de Nicoya, Costa Rica, para conocer su estado actual de explotación y caracterizar normas fundamentales para el manejo de esta pesquería.

\section{MATERIALES Y MÉTODOS}

Los 2831 especímenes de la población del complejo Opisthonema en el Golfo de Nicoya, Costa Rica, fueron recolectados mensualmente, entre junio del 2007 y mayo del 2008, en la parte externa del
Golfo de Nicoya $\left(09^{\circ} 52^{\prime} \mathrm{N}\right.$ y $\left.84^{\circ} 45^{\prime} \mathrm{W}\right)$ a bordo del buque Cap. Villa.

Para asegurar una buena distribución de las frecuencias de las tallas capturadas, las muestras fueron obtenidas de submuestras recolectadas al azar de cada uno de los lances realizados durante el viaje de pesca.

Los especímenes fueron clasificados taxonómicamente por medio de la clave propuesta por Berry y Barrett (1963); se les registró la longitud total (LT) y estándar (LS) en centímetros con $\pm 0.05 \mathrm{~cm}$ de precisión y el peso total (PT) y de la gónada (PG) en gramos con $\pm 0.005 \mathrm{~g}$ de precisión.

La identificación por sexo se realizó mediante disección y caracterización gonádica, su desarrollo fue determinado utilizando la escala macroscópica propuesta por Rodríguez et al. (1986) y modificada por Vega-Cendejas et al. (1997):

- Estadio I: Sardina indefinida, ausencia de gónadas, no es posible definir el sexo, se presenta en individuos jóvenes.

- Estadio II: Sardina virgen, gónadas pequeñas, huevos no visibles a simple vista, ovarios de color blanco o rojizo claro; testículos de blanquecinos a rojizos o grisáceos.

- Estadio III: Sardina madura, las gónadas ocupan casi la mitad del cuerpo, de 2 a $3 \mathrm{~cm}$ de largo, los huevos visibles son relativamente pocos. Ovario de color vino, rojizo o naranja, en los casos de reabsorción se pueden ver vasos sanguíneos prominentes; testículos grisáceos.

- Estadio IV: Sardina en desove, gónada grande, huevos observables a simple vista, estos son redondos, opacos o transparentes, de color naranja o amarillento; testículos blancos en diferentes tonalidades.

- Estadio V: Sardina desovada, gónada grande en forma de bolsa ensangrentada, ovarios vacíos o con huevos residuales; 
testículos largos y flácidos, pueden contener remanentes de esperma.

La época de máximo desove para $O$. libertate se obtuvo al comparar los máximos valores mensuales del índice gonadosomático: $\mathrm{IGS}=(\mathrm{PT} * \mathrm{PG}) / 100 \quad(\mathrm{Gar}-$ cía-Abad et al. 1998), con la abundancia por mes de individuos maduros ( $\geq$ estadio III). Esta época fue comparada con los datos medios mensuales de precipitación y temperatura ambiental de la Estación Meteorológica de Puntarenas $\left(09^{\circ} 58^{\prime} \mathrm{N} \mathrm{y}\right.$ $84^{\circ} 50^{\prime} \mathrm{W}$; elevación $3 \mathrm{~m}$ ), para el período de muestreo, facilitada por el Instituto Meteorológico Nacional de Costa Rica.

La talla media de primera madurez sexual $\left(\mathrm{TPM}_{50 \%}\right)$ de las hembras de $O$. libertate se obtuvo estimando la fracción de ejemplares maduros ( $\geq$ estadio III) en cada intervalo de talla, mediante la siguiente ecuación de la curva logística: $\mathrm{FM}_{(\mathrm{L})}=1 / 1+\mathrm{e}^{\left(\mathrm{S} 1-\mathrm{S} 2^{*} \mathrm{~L}\right)}$. Donde: $\mathrm{FM}_{(\mathrm{L})}$ es la proporción de individuos maduros en cada talla; $\mathrm{S}_{1}$ y $\mathrm{S}_{2}$ son constantes del modelo; L es la marca de clase de la longitud de referencia. Para calcular $\mathrm{S}_{1}$ y $\mathrm{S}_{2}$ se usó la ecuación de la regresión lineal: $\operatorname{Ln}\left(1 / \mathrm{F}_{(\mathrm{L})}-1\right)=\mathrm{S}_{1}-\mathrm{S}_{2} * \mathrm{~L}$. Donde: $\mathrm{F}_{(\mathrm{L})}$ es la fracción de especímenes maduros $(\geq$ estadio III) en cada intervalo de talla; $L$ es la marca de clase; $\mathrm{S}_{1}=$ a y $\mathrm{S}_{2}=-$ b. $\mathrm{La} \mathrm{TPM}_{50 \%}=\mathrm{S}_{1} / \mathrm{S}_{2}$ (Gassman et al. 2008).

Las frecuencias de longitud de $O$. libertate y $O$. medirastre fueron analizadas por medio del paquete estadístico FiSAT (FAO-IOCLARM Stock Assessment Tool) (Gayanilo y Pauly, 1994), para obtener los parámetros de crecimiento para la ecuación de von Bertalanffy: $L t=L_{\infty}\left[1-\exp ^{-k(t-0)}\right]$. Donde: $\mathrm{Lt}=$ longitud del pez a la edad " t"; $\mathrm{L}_{\infty}=$ constante, longitud asintótica (cm) (longitud que el pez puede alcanzar cuanto $t \rightarrow \infty$ ); $K=$ constante, coeficiente de crecimiento; $\mathrm{t}=$ edad (años); $\mathrm{t}=$ representa la edad del pez (años) cuando su longitud es igual a cero; este parámetro fue estimado por medio de la ecuación de Pauly: $\log \left(-\mathrm{t}_{\mathrm{o}}\right)=-0.3922-\left(0.2752 \log \mathrm{L}_{\infty}(\mathrm{cm})\right)-$ (1.038LogK/año).

Para obtener mayor confiabilidad, los parámetros de crecimiento obtenidos fueron comparados con los resultados reportados por otros autores, mediante el índice de crecimiento estándar "phi intraespecífico", $\varphi=\log \mathrm{K}+2 \log \mathrm{L}_{\infty}$.

La edad límite $\left(\mathrm{A}_{0.95}\right)$ fue calculada según el criterio de Taylor (González-Cabellos y Mengual-Izquierdo, 1995): $\mathrm{A}_{0.95}=$ $\mathrm{t}_{\mathrm{o}}+2.996 / \mathrm{K}$. Donde: $\mathrm{A}_{0.95}=$ edad límite del tiempo requerido para alcanzar el $95 \%$ de $\mathrm{L}_{\infty}$ Para obtener los patrones de reclutamiento se graficaron, por medio de FiSAT, las frecuencias de longitud por retrocálculo sobre el tiempo (Gayanilo y Pauly, 1994).

La mortalidad natural (M) y la total (Z) fueron calculadas por medio de FiSAT. Z utilizando el método de Beverton y Holt y M mediante la fórmula empírica de Pauly: $\ln \mathrm{M}=\quad-0.0152-0.279 * \operatorname{lnL}_{\infty}+0.6543 * 1-$ $\mathrm{nK}+0.463 * \ln \mathrm{T}$ (Sparre y Venema, 1995). Donde la $\mathrm{T}=28^{\circ} \mathrm{C}$, temperatura media anual descrita para el Golfo de Nicoya, con pocas variaciones a través del año (Valdés et al. 1987, citado en Soto, 1999). De esta manera, obteniendo $Z$ y $M$ se calculó la mortalidad por pesca (F), a través de $\mathrm{Z}=\mathrm{F}+\mathrm{M}$, lo cual permite determinar el coeficiente de explotación (E) de la siguiente manera: $E=F / Z$ (Gulland, 1971).

Finalmente, se determinaron la frecuencia acumulada de tallas de $O$. libertate y el porcentaje de especímenes y biomasa que están siendo capturados antes de alcanzar la $\mathrm{TPM}_{50 \%}$ estimada.

\section{RESULTADOS}


O. libertate es la especie más abundante (78.4\%) de los 2831 especímenes del complejo Opisthonema analizados de la captura en el Golfo de Nicoya, Costa Rica, seguida por $O$. medirastre $(16.9 \%)$ y O. bulleri (4.7\%) (Cuadro 1).

La poca representatividad de $O$. medirastre y $O$. bulleri, en porcentaje de abundancia $\mathrm{y}$ discontinuidad en aparición mensual durante el año de muestreo, hace que los parámetros de crecimiento de la ecuación de von Bertalanffy, la edad límite y las tasas de mortalidad estimadas para $O$. medirastre sean poco confiables, en tanto que para $O$. bulleri no se pudieron realizar, debido a que existía alta probabilidad de obtener un sesgo importante entre el valor estimado y el verdadero (Cuadro 1 y Fig. 1).

La elevada media del IGS $(\approx 1.5)$ y la alta prevalencia $(>50 \%)$ de hembras con gónadas maduras (III-V) encontradas, durante los meses de febrero, marzo, abril y mayo, demuestran el período con mayor intensidad de desove para $O$. libertate en el área de estudio (Fig. 2 y Fig. 3).

Esta época de máximo desove muestra correspondencia con los meses que presentan menor precipitación y máxima temperatura ambiental (Fig. 4).

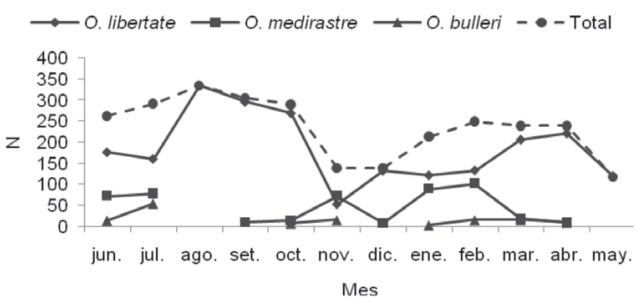

Fig. 1. Número de especímenes muestreados por especie del complejo Opisthonema de las capturas por mes en el Golfo de Nicoya, Costa Rica

Fig. 1. Number of organisms by specie, accord into to the catch by month of Opisthonema in Golfo de Nicoya, Costa Rica
La proporción de especímenes maduros ( $\geq$ estadio III) por frecuencia de talla se muestra en el cuadro 2. Las constantes de la ecuación logística estimadas por la regresión lineal para $O$. libertate correspondieron a: $\mathrm{S}_{1}=16.254$ y $\mathrm{S}_{2}=0.8703$, y según la ecuación obtenida de la función logística de mejor ajuste, la $\mathrm{TPM}_{50 \%}$ para esta especie es alcanzada a los $18.68 \mathrm{~cm}$ de LT (Fig. 5).

La aproximación entre los valores $\varnothing$, reportados por otros autores en diferentes localidades, valida los parámetros poblacionales de la ecuación de von Bertalanffy, estimados para $O$. libertate y $O$. medirastre. Este índice presenta, para cada una de las especies, mayor similitud con respecto a la misma área de estudio. $O$. libertate muestra una disminución en la $\mathrm{L}_{\infty}$ (Cuadro 3).

Las curvas de crecimiento en longitud, para $O$. libertate y $O$. medirastre, describen un crecimiento acelerado en el primer año de edad para cada una de las especies (Fig. 6). La $\mathrm{A}_{0.95}$ calculada para O. libertate fue igual a 2.78 años y para $O$. medirastre de 2.57 años; luego de esta talla, la tasa de crecimiento es cercana a cero. De acuerdo con la ecuación de crecimiento en longitud, la $\mathrm{TPM}_{50 \%}$ estimada para

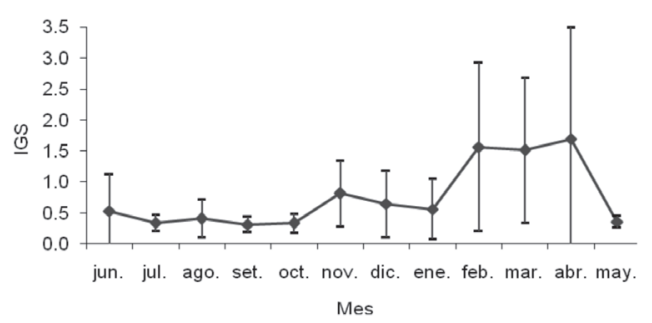

Fig. 2. Media del índice gonadosomático mensual \pm D.S. para las hembras de $O$. libertate en el Golfo de Nicoya, Costa Rica

Fig. 2. Monthly gonadosomatic index $\pm \mathrm{SD}$ of female O. libertate in Golfo de Nicoya, Costa Rica 
Cuadro 1. Número de especímenes por especie del complejo Opisthonema y sus respectivos límites de longitud total y peso total muestreados de la captura en el Golfo de Nicoya, Costa Rica

Table 1. Number of organisms by specie and their length total and weight total limits, according to the catch of Opishtonema in Golfo de Nicoya, Costa Rica

\begin{tabular}{lccc}
\hline & \multicolumn{3}{c}{ Especie } \\
\cline { 2 - 4 } & O. libertate & O. medirastre & O. bulleri \\
\hline $\mathrm{N}$ & 2220 & 478 & 133 \\
$\mathrm{LT}_{(\max )}$ & 25.6 & 31.2 & 29.7 \\
$\mathrm{LT}_{(\min )}$ & 10.6 & 11 & 13.4 \\
$\mathrm{PT}_{(\max )}$ & 169.02 & 304.24 & 264.55 \\
$\mathrm{PT}_{(\min )}$ & 11.29 & 12.95 & 20.87 \\
\hline
\end{tabular}

$\mathrm{N}=$ número de especímenes; $\mathrm{LT}=$ longitud total $(\mathrm{cm}) ; \mathrm{PT}=$ peso total $(\mathrm{g})$.
Cuadro 2. Fracción de especímenes maduros en estadio III-V de $O$. libertate en cada intervalo de talla, durante el período de muestreo en el Golfo de Nicoya, Costa Rica Table 2. O. libertate mature specimens in stage III-V in each length interval during the sampling period in Golfo de Nicoya, Costa Rica

\begin{tabular}{cc}
\hline $\mathbf{L}$ & $\mathbf{F}_{(\mathbf{L})}$ \\
\hline 14.5 & 0.000 \\
15.5 & 0.070 \\
16.5 & 0.109 \\
17.5 & 0.238 \\
18.5 & 0.508 \\
19.5 & 0.667 \\
20.5 & 0.990 \\
21.5 & 1.000 \\
\hline $\mathrm{F}_{(\mathrm{L})}=$ fracción de especímenes maduros; \\
$\mathrm{L}=$ intervalo de talla.
\end{tabular}

Cuadro 3. Parámetros de crecimiento de la ecuación de von Bertalanffy para el complejo Opisthonema, estimados por diferentes autores

Table 3. von Bertalanffy growth parameters determined by different authors for Opisthonema

\begin{tabular}{|c|c|c|c|c|c|c|c|c|}
\hline Fuente & Localidad & Especie & & $\begin{array}{c}\mathbf{L}_{\infty} \\
(\mathbf{c m})\end{array}$ & $\begin{array}{c}\mathbf{K} \\
\left(\text { (años }^{-1}\right)\end{array}$ & $\begin{array}{c}\mathbf{t}_{0} \\
\text { (años) }\end{array}$ & $\varnothing$ & Talla \\
\hline Fuentes (1983) & Ecuador & Opisthonema $s p_{t}$ & & 31.7 & 0.216 & -2.52 & 2.34 & LT \\
\hline $\begin{array}{l}\text { Gallardo et al. } \\
\quad \text { (1993) }\end{array}$ & Golfo California Sur & O. libertate & & 20.8 & 0.3923 & -1.381 & 2.23 & LS \\
\hline $\begin{array}{c}\text { Carmona y } \\
\text { Alexandres } \\
(1994)\end{array}$ & $\begin{array}{l}\text { Bahía Magdalena, } \\
\text { California Sur }\end{array}$ & O. libertate & & 19.4 & 0.812 & -0.015 & 2.49 & LS \\
\hline $\begin{array}{c}\text { Soto y Rodríguez } \\
\text { (1999) }\end{array}$ & $\begin{array}{l}\text { Pacífico } \\
\text { Costa Rica }\end{array}$ & O. medirastre & $\begin{array}{l}\mathrm{H} \\
\mathrm{M}\end{array}$ & $\begin{array}{l}30.6 \\
28.0\end{array}$ & $\begin{array}{c}0.96 \\
1.2\end{array}$ & $\begin{array}{l}-0.16 \\
-0.13\end{array}$ & $\begin{array}{l}2.95 \\
2.97\end{array}$ & LT \\
\hline Soto (1999) & $\begin{array}{l}\text { Golfo Nicoya, } \\
\text { Costa Rica }\end{array}$ & $\begin{array}{l}\text { O. libertate } \\
\text { O. bulleri }\end{array}$ & $\begin{array}{l}\mathrm{H} \\
\mathrm{M} \\
\mathrm{H} \\
\mathrm{M}\end{array}$ & $\begin{array}{l}30.0 \\
30.1 \\
29.8 \\
29.5\end{array}$ & $\begin{array}{l}1.6 \\
1.1 \\
1.1 \\
1.4\end{array}$ & $\begin{array}{l}-0.10 \\
-0.14 \\
-0.15 \\
-0.11\end{array}$ & $\begin{array}{l}3.16 \\
3.00 \\
2.99 \\
3.09\end{array}$ & LT \\
\hline $\begin{array}{l}\text { Rodríguez et al. } \\
\text { (1999) }\end{array}$ & $\begin{array}{l}\text { Golfo Nicoya, } \\
\text { Costa Rica }\end{array}$ & $\begin{array}{l}\text { O. libertate } \\
\text { O. medirastre } \\
\text { O. bulleri }\end{array}$ & & $\begin{array}{l}36.0 \\
34.6 \\
31.0\end{array}$ & $\begin{array}{l}1.1 \\
1.1 \\
0.7\end{array}$ & $\begin{array}{l}-0.14 \\
-0.13 \\
-0.22\end{array}$ & $\begin{array}{l}3.15 \\
3.12 \\
2.83\end{array}$ & LT \\
\hline Este trabajo & $\begin{array}{l}\text { Golfo Nicoya, } \\
\text { Costa Rica }\end{array}$ & $\begin{array}{l}\text { O. libertate } \\
\text { O. medirastre }\end{array}$ & & $\begin{array}{l}25.9 \\
32.0\end{array}$ & $\begin{array}{l}1.03 \\
1.10\end{array}$ & $\begin{array}{l}-0.13 \\
-0.15\end{array}$ & $\begin{array}{l}2.84 \\
3,05\end{array}$ & LT \\
\hline
\end{tabular}

$\mathrm{H}=$ hembras; $\mathrm{M}=$ machos; $\mathrm{LT}=$ longitud total; $\mathrm{LS}=$ longitud estándar. 

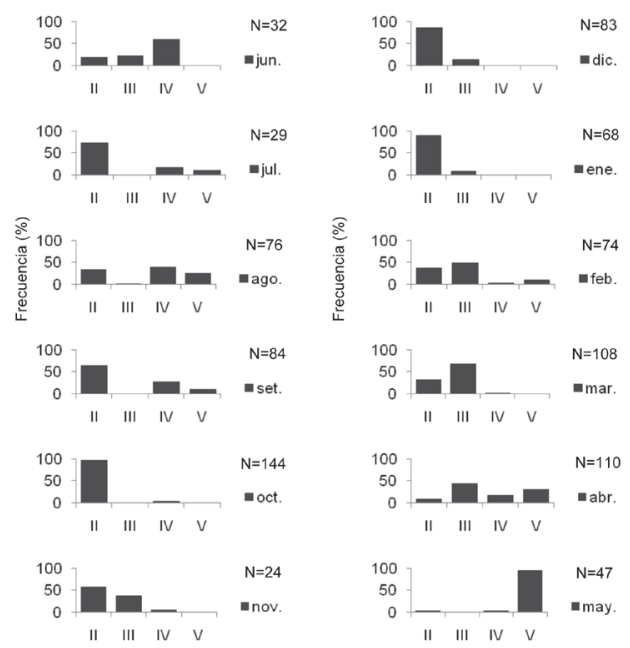

Fig. 3. Porcentaje de frecuencia mensual de los estados de madurez en hembras de $O$. libertate en el Golfo de Nicoya, Costa Rica

Fig. 3. Monthly percentage frequency of the maturity stages in female $O$. libertate in Golfo de Nicoya, Costa Rica

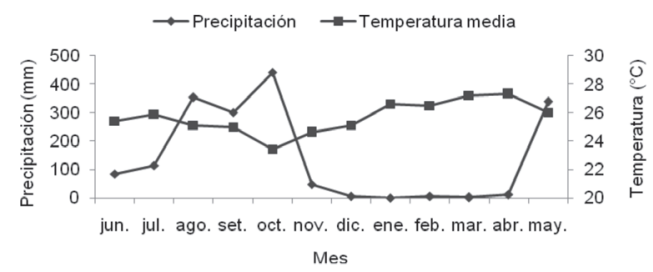

Fig. 4. Precipitación acumulada y temperatura media mensual ambiental para los meses de muestreo de la Estación Meteorológica de Puntarenas, Costa Rica. Fuente: Instituto Meteorológico Nacional, Costa Rica

Fig. 4. Monthly accumulated precipitation and environmental average temperature during the sampling period of Estación Meteorológica, Puntarenas, Costa Rica. Taked from: Instituto Meteorológico Nacional, Costa Rica
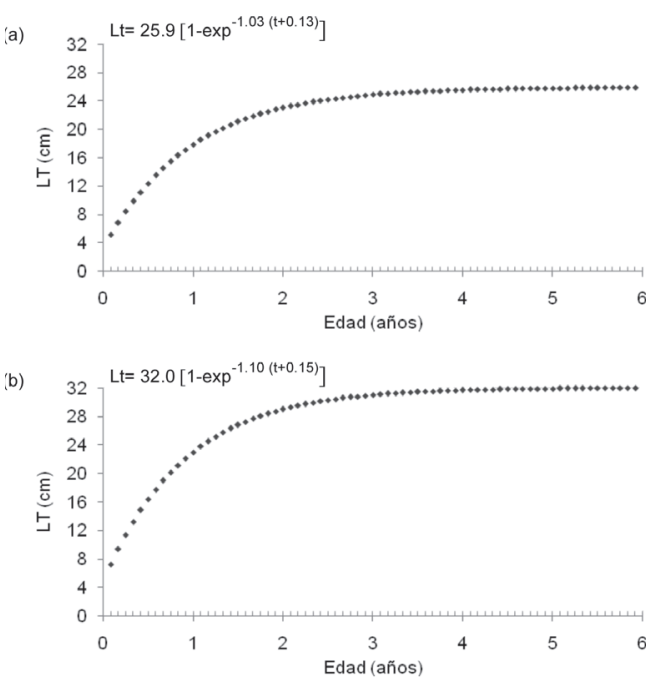

Fig. 6. Curva de crecimiento en longitud para a) $O$. libertate y b) $O$. medirastre, en el Golfo de Nicoya, Costa Rica

Fig. 6. Growth cruved based in length for a) $O$. libertate and b) O. medirastre in Golfo de Nicoya, Costa Rica 
Cuadro 4. Tasas de mortalidad y coeficiente de explotación para el stock explotable de $O$. libertate y $O$. medirastre en el Golfo de Nicoya, Costa Rica

Table 4. Mortality rate and exploitation ratio of the exploitable stock of $O$. libertate and $O$. medirastre in Golfo de Nicoya, Costa Rica

\begin{tabular}{lcccc}
\hline Especie & $\mathbf{M}$ & $\mathbf{F}$ & $\mathbf{Z}$ & $\mathbf{E}$ \\
\hline O. libertate & 1.89 & 3.55 & 5.44 & 0.65 \\
O. medirastre & 1.86 & 2.31 & 4.17 & 0.55 \\
\hline
\end{tabular}

$\mathrm{M}=$ mortalidad natural; $\mathrm{F}=$ mortalidad por pesca; $\mathrm{Z}=$ mortalidad total; $\mathrm{E}=$ índice de explotación.

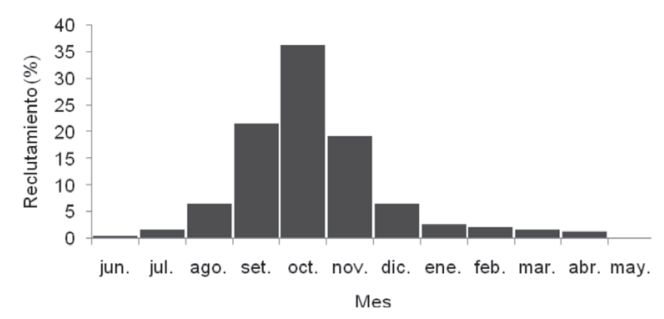

Fig. 7. Patrón de reclutamiento para O. libertate en el Golfo de Nicoya, Costa Rica

Fig. 7. Recruitment patterns for $O$. libertate in Golfo de Nicoya, Costa Rica

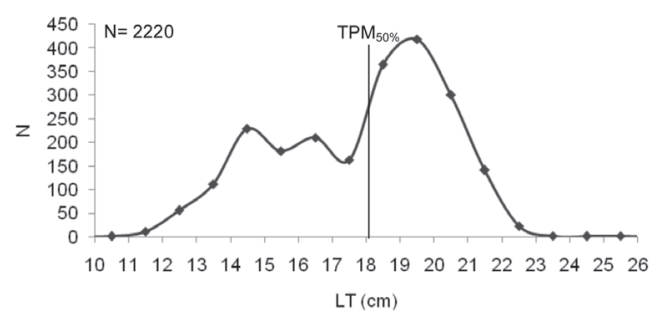

Fig. 8. Frecuencia de tallas de la captura de $O$. libertate en el Golfo de Nicoya, Costa Rica

Fig. 8. Length frecuency of $O$. libertate catch in Golfo de Nicoya, Costa Rica
O. libertate se alcanza a los 13.32 meses de edad y la talla mínima de captura permitida para esta pesquería (15 cm de LT) (INCOPESCA, 1998) a los 8.52 meses.

El patrón de reclutamiento presentó una sola moda entre septiembre y noviembre, donde se concentró más del 75\% de los reclutas, con la máxima intensidad en octubre (Fig. 7), mes que presenta la mayor precipitación y la menor temperatura ambiental en el área de estudio (Fig. 4).

Las tasas de mortalidad natural para ambas poblaciones $(O$. libertate y $O$. medirastre) son muy similares, sin embargo, presentan variación en la mortalidad por pesca. Ambos stock se encuentran en un estado de sobreexplotación (Cuadro 4).

La distribución de la frecuencia de tallas acumuladas de la captura de los especímenes de $O$. libertate en el Golfo de Nicoya, Costa Rica, se muestra en la figura 8.

De acuerdo con la $\mathrm{TPM}_{50 \%}$ definida para $O$. libertate, el $43 \%$ de los especímenes de esta especie están siendo capturados antes de alcanzar su talla media de reproducción, lo cual corresponde al 25\% de la biomasa total capturada.

\section{DISCUSIÓN}

La composición por especie obtenida presenta una gran relación con lo reportado por Berry y Barrett (1963), a partir de 19 recolecciones hechas en el Golfo de Nicoya, entre los años 1952 y 1960, cuando la pesquería de sardina no había iniciado en el país. Al igual que en el Golfo de California, O. libertate es la especie del complejo Opisthonema que presenta el mayor aporte en las capturas (Ruiz y Lyle, 1992). O. bulleri, en las costas de Sinaloa y Nayarit, México, también es la especie que ocupa el tercer lugar en las capturas 
de este género (Castro-González et al. 1997). Esto sugiere que la composición por especie en las capturas del complejo Opisthonema, en el área de estudio, refleja la abundancia por especie en el medio ambiente. Además, indica, como mencionan Stevenson y Carranza (1981), que esta pesquería está destinada a la captura simultánea de las tres especies de este género y no existe evidencia para determinar que sea selectiva.

La discontinuidad en la aparición mensual de $O$. medirastre y $O$. bulleri, en las capturas de este complejo en el Pacífico de Costa Rica, ha sido reportada anteriormente (Rodríguez et al. 1999; Soto, 1999). Esta condición y la abundancia por especie posiblemente estén determinadas por alguna condición ecológica, establecida sobre todo por la temperatura (López-Lemus, 1993). Soto (1999) y Castro-González et al. (1997) atribuyen la variabilidad temporal de aparición y la poca abundancia de $O$. bulleri en aguas someras, a que su hábitat es más oceánico.

La presencia de varios estadios de madurez, durante la mayoría de los meses del año de muestreo, evidencian que $O$. libertate se reproduce durante todo el año, lo cual refuerza las observaciones de Peterson (1956), Rodríguez et al. (1986) y Stevenson y Carranza (1981). El análisis del IGS mostró que el período máximo de desove para esta especie se presenta durante los meses más calurosos del año. García-Abad et al. (1998), Funes-Rodríguez et al. (2001) y Moser et al. (1974), han definido que las especies del género Opisthonema desovan predominantemente cuando la temperatura de la superficie del mar alcanza sus máximos en verano.

Funes-Rodríguez et al. (2001) describen que la época de desove de los pequeños pelágicos probablemente esté condicionada, para que cuando ocurra el afloramiento estacional del plancton las larvas ya estén presentes. De forma semejante, el desove de $O$. libertate, en el Golfo de Nicoya, precede a los meses de máxima concentración de microfitoplancton (junio-julio) para el área de estudio, según los datos facilitados por el Laboratorio de Fitoplancton Marino de la Estación de Biología Marina de la Universidad Nacional, Costa Rica.

Lo anterior evidencia que los períodos de veda definidos para esta pesquería, en los años 2007 y 2008 (INCOPESCA, 2007, 2008), se encontraban desfasados con respecto al período de máxima reproducción para la especie de mayor abundancia en esta pesquería.

La $\mathrm{TPM}_{50 \%}$ calculada para $O$. libertate presenta una gran relación con las estimaciones de este parámetro realizadas para la misma población durante la temporada 1985-1986 (Soto, 1999) y para la misma especie en la zona de Mazatlán, México (Páez-Barrera, 1976). Sin embargo, se encuentra por encima de la $\operatorname{TPM}_{50 \%}(11$ cm de LT) y de la talla de primera captura permitida (15 cm de LT) (INCOPESCA, 1998). Esta situación demuestra un error de ordenación para esta pesquería en la legislación nacional, debido posiblemente a una inadecuada interpretación en la información técnica suministrada.

Stevenson y Carranza (1981) definieron al complejo Opisthonema en la costa pacífica de Costa Rica como una sola unidad geográfica de stock. Esto debido a que no existe pesquería de este recurso en ningún otro país de América Central, solamente en Panamá, donde la flota pesquera se concentra en el Golfo de Panamá y no se desplaza hacia el límite con Costa Rica, lo que permite considerar que este estudio está basado en el análisis de una sola comunidad formada por las poblaciones de 
cada una de las especies simpátricas que constituyen el complejo Opisthonema.

El Ø' de O. libertate y $O$. medirastre mostró similitud entre los parámetros de crecimiento obtenidos y los reportados para estas especies en la misma área de estudio, durante la temporada de 1985-1986 (Soto, 1999) y 1998-1999 (Rodríguez et al. 1999), lo que genera una alta confiabilidad de los parámetros estimados. La disminución en la $\mathrm{L}_{\infty}$ de $O$. libertate puede responder a las variaciones propias del esfuerzo pesquero, o bien, a los cambios ambientales sufridos por la especie (Csirke, 1980). Las variaciones de $\varnothing$ ', con respecto a los demás autores, podrían ser debidas a las diferencias en el crecimiento de estas especies por habitar en otras latitudes con distintas características ambientales.

La curva de crecimiento para $O$. libertate, obtenida a partir de los parámetros poblacionales de la ecuación de von Bertalanffy, muestra una relación directa entre la época de desove, el tiempo en que tarda la especie para alcanzar la talla mínima de captura permitida y la época de reclutamiento, ya que el desove inicia en febrero y 8.52 meses después (octubre) se obtiene el máximo reclutamiento. Esta situación es considerada como normal, al tratarse de una especie de ciclo de vida corto, condición que fue validada por medio de la edad límite estimada para la especie.

El período de máximo reclutamiento se establece durante la época de lluvias, coincidiendo con lo reportado por Rodríguez et al. (1999) y con las observaciones de García-Abad et al. (1998). La presencia de prerreclutas durante la mayoría de los meses muestreados, junto con la observación de cardúmenes de juveniles, en los meses de julio, agosto, septiembre y octubre, además de la captura y liberación de 23 TM (según el Capitán) de sardina por debajo de la talla mínima de captura permitida en septiembre, confirman la época de reclutamiento obtenida y comprueban que el reclutamiento de esta población al stock explotable, al igual que su reproducción, son continuos durante todo el año (Peterson, 1956; Rodríguez et al. 1986, 1989, 1999; Stevenson y Carranza, 1981).

Las altas tasas de mortalidad natural estimadas para $O$. libertate y $O$. medirastre son justificables, por tratarse de especies de ciclo de vida corto y por ser altamente depredadas, al representar el alimento de un gran número de peces, moluscos y aves (Gallardo-Cabello et al. 1991; Mexicano-Cíntora et al. 1996), constituyéndose como organismos de gran importancia en la red alimenticia de las aguas del Golfo de Nicoya.

La gran importancia de estas especies para la pesquería de cerco y la industria y su utilización como carnada, sumada a la gran proporción de especímenes capturados antes de que alcancen su TPM $_{50 \%}$, hace que su mortalidad por pesca sea mayor que la natural.

La diferencia en las tasas de mortalidad por pesca entre $O$. libertate y $O$. medirastre, a pesar de que ambas especies son igualmente susceptibles a la captura, es aceptable, debido a que son poblaciones genéticamente diferentes (Dennis y López-Lemus, 1988), con estrategias ecológicas distintas.

$O$. libertate, al ser la especie más abundante en las capturas del complejo Opisthonema en el Golfo de Nicoya, muestra un mayor nivel de explotación que $O$. medirastre. Sin embargo, al comparar estos resultados con los definidos por Rodríguez et al. (1999), para la misma población durante 1998-1999, se nota una mejoría en el índice de explotación para ambas especies, posiblemente debida a que la cantidad de esfuerzo de pesca que 
se ha mantenido hasta el momento le ha permitido al stock ir alcanzando un nuevo punto de equilibrio entre la mortalidad por pesca y la natural.

Para el manejo de esta pesquería se recomienda no incrementar el esfuerzo pesquero y establecer dos vedas al año; una durante la época de máxima reproducción, entre febrero y mayo, para proteger la biomasa desovante, y la otra durante el mes de octubre, para proteger la biomasa virgen.

\section{AGRADECIMIENTOS}

A Blue Riviera and Co. Ltda., por aportar las muestras para el estudio y permitir la observación a bordo de su buque sardinero Cap. Villa. Al Instituto Meteorológico Nacional, por proporcionar los datos de los parámetros ambientales y a la Estación de Biología Marina de la Universidad Nacional, por proporcionar la infraestructura y el equipo necesario para los análisis. Al Programa de Maestría en Ciencias Marinas y Costeras de la Universidad Nacional de Costa Rica y a Mauricio Vargas Barquero, Rosa Soto Rojas y Luis Villalobos Chacón, por el asesoramiento durante la investigación. Este estudio fue posible gracias al financiamiento del CONICIT y Sardimar S.A.

\section{BIBLIOGRAFÍA}

Berry, H. \& Barrett, I. (1963). Guillraker analysis and speciation in the thread hering genus Opisthonema. Inter. Amer. Trop. Tuna. Comm. Bull., VII, 113-190.

Bussing, W. \& López, M. (1993). Peces demersales y pelágicos costeros del Pacífico de Centroamérica meridional. Guía ilustrada. San José, Costa Rica. Rev. Biol. Trop. (publicación especial), 7-164.
Carmona, R. \& Alexandres, F. (1994). Determinación del crecimiento de Opisthonema libertate (Clupeiformes: Clupeidae) mediante lectura de otolitos. Rev. Biol. Trop., 42, 233-238.

Castro-González, J. J., Lizárraga-Rodríguez, H. M. \& Chapa-Morales, O. (1997). Fecundidad de la sardina crinuda Opisthonema bulleri (Pisces: Clupeidae) entre las costas de Sinaloa y Nayarit, México. Rev. Biol. Trop., 44/45, 659-661.

Csirke, J. (1980). Introducción a la dinámica de poblaciones de peces. FAO. Doc. Téc. Pesca 92. Roma, Italia: FAO.

Dennis, K. \& López-Lemus, L. (1988). Biochemical genetic and morphological divergence among three species of thread herring (Opisthonema) in northwest México. CalCOFI Rep. XXIX, 110-121.

Fuentes, E. (1983). Estudio preliminar de edad y crecimiento de Opisthonema spp. en aguas ecuatorianas. Rev. Cien. Mar. Limn., 2, 109-114.

Funes-Rodríguez, R., Hinojosa-Medina, A., Avendaño-Ibarra, R., HernándezRivas, M., Saldiera-Martínez, R. \& Watson, W. (2001). Spawning of small pelagic fishes in Bahía Magdalena, Baja California Sur, México, at the beninning of the 1997-1998 El Niño event. Estuar. Coast. Shelf Sci., 53, 653-664.

Gallardo, M., Laguarda, A. \& Corrales, R. (1993). Análisis de la edad, crecimiento y mortalidad natural de la sardina crinuda Opisthonema libertate (Gunther, 1868) de las aguas del sur del Golfo de California. Ciencia Pesquera, 9, 137-146.

Gallardo-Cabello, M., Jacob-Cervantes, M. \& Chiappa-Carrara, X. (1991). Análisis de los hábitos alimentarios y del aporte 
del carbono de las presas a la dieta de la sardina crinuda, Opisthonema libertate, Gunther 1866 (Pisces: Clupeidae) en el Golfo de California. An. Inst. Cienc. del Mar y Limnol., 18, 37-48.

García-Abad, M. C., Yáñez-Arancibia, A., Sánchez-Gil, P. \& Tapia-García, M. (1998). Distribución, abundancia y reproducción de Opisthonema oglinum (Pisces: Clupeidae) en la plataforma continental del sur del Golfo de México. Rev. Biol. Trop., 46, 257-266.

Gassman, J., Esclava, N. \& González, L. (2008). Reproducción de la sardina, Sardinella auriata (Clupeiformes: Clupeidae) del sureste de la Isla de Margarita, Venezuela. Rev. Biol. Trop., 56, 1813-1824.

Gayanilo, F. \& Pauly, D. (1994). The FAOICLARM stock assessment, tools (FiSAT). User Guide. Roma, Italia: FAO.

González-Cabellos, L. \& Mengual-Izquierdo, A. (1995). Edad y crecimiento del machuelo, Opisthonema oglinum (Le Sueur, 1818) (Teleostei: Clupeidae), de la Isla Margarita, Venezuela. Ciencias Marinas, 21, 387-399.

Gulland, J. (1971). Manual de métodos para la evaluación de las poblaciones de peces. Manuales en Ciencia Pesquera 4. Roma, Italia: FAO.

INCOPESCA. (1998). Prohíbe captura de especimenes juveniles de sardina gallera. Acuerdo 215. (Publicado en La Gaceta $\mathrm{N}^{\circ} 172,3$ de septiembre). San José, Costa Rica: Imprenta Nacional.

INCOPESCA. (2007). Decreta periodo de veda en el Golfo de Nicoya. Acuerdo 074. (Publicado en La Gaceta $\mathrm{N}^{\circ}$ 58, 22 de marzo). San José, Costa Rica: Imprenta Nacional.

INCOPESCA. (2008). Decreta periodo de veda para el Golfo de Nicoya co- rrespondiente al año 2008. Acuerdo 137. (Publicado en La Gaceta $\mathrm{N}^{\circ} 79$, 24 de abril). San José, Costa Rica: Imprenta Nacional.

López-Lemus, L. G. (1993). Diferencias termo-adaptativas de enzimas deshidrogenasas NAD-dependientes de peces marinos pelágicos templados y tropicales. Ciencia Pesquera, 10, 89-95.

Mexicano-Cíntora, G., Salas, S. \& Cabrera, M. A. (1996). Análisis de la pesquería y dinámica poblacional de la vivita de hebra (Opisthonema oglinum) en Yucatán, México. Ciencias Marinas, 22, 9-22.

Moser, H. G., Ahlstrom, E. H., Kramer, D. \& Stevens, E. G. (1974). Distribution and abundance of fish eggs and larvae in the Gulf of California. Calif. Coop. Oceanic Fish. Invest. Rep., 17, 112-130.

Páez-Barrera, F. (1976). Desarrollo gonadal, madurez, desove y fecundidad de sardina crinuda, Opisthonema libertate (Günther) de la zona de Mazatlán, basados en el análisis histológico de la gónada. Memoria del Simposium sobre Recursos Masivos de México (28-30 de septiembre de 1976). Ensenada, B.C. México.

Peterson, C. (1956). Observations on the taxonomy, biology and ecology of the engraulid and clupeid fishes in the Gulf of Nicoya, Costa Rica. Int. Amer. Trop. Tuna Comm. Bull., 1, 139-280.

Rodríguez, J., Palacios, J. A. \& Chavarría, A. (1986). Fecundidad y época de desove del arenque hebra Opisthonema libertate (Pisces: Clupeidae) en el Golfo de Nicoya, Costa Rica. Uniciencia, 3, 87-93.

Rodríguez, J., Palacios, J. A. \& Chavarría, A. (1989). Época de maduración y fecundidad de la sardina gallera Opisthonema medirastre y $O$. bulleri 
(Pisces: Clupeidae) en la costa pacífica central de Costa Rica. Rev. Biol. Trop., 37, 49-54.

Rodríguez, J., Soto, R. \& Palacios, J. A. (1999). Evaluación del stock explotable de la sardina Opisthonema spp. por la flota cerquera del Pacifico Central. Informe Científico Técnico no publicado. Universidad Nacional, Costa Rica.

Ruiz, L. \& Lyle, F. (1992). Fluctuaciones periódicas de la captura de sardina crinuda (Opisthonema spp.) en el Golfo de California, 1972-1990. CalCOFI Rep., 33, 124-129.

Soto, R. (1999). Estimación de la curva de crecimiento y talla de la primera madurez de tres especies del complejo Opisthonema (Pisces: Clupeidae) en el Golfo de Nicoya, Costa Rica. Tesis de Licenciatura no publicada. Universidad Nacional, Costa Rica.

Soto, R. \& Rodríguez, J. (1999). Dinámica poblacional de Opisthonema medirastre (Pisces: Clupeidae) en la costa pacífica de Costa Rica. Uniciencia, 15 16, 61-64.

Sparre, P. \& Venema, S. (1995). Introducción a la evaluación de recursos pesqueros tropicales. FAO Doc. Téc. Pesca 306/1. Roma, Italia: FAO.
Stevenson, D. \& Carranza, F. (1981). Maximum yield estimates for the pacific thread herring, Opisthonema spp., fishery in Costa Rica. Fish. Bull., 79, 689-703.

Vega-Cendejas, M., Mexicano-Cíntora, G. \& Arce, A. (1997). Biology of the thread herring Opisthonema oglinum (Pisces: Clupeidae) from a beach seine fishery of the Campeche Bank, Mexico. Fish. Res., 30, 117-126.

Víquez, R. (1990). Algunos aspectos biométricos e indices relativos de crecimiento, de 3 especies del género Opisthonema (Pisces: Clupeidae) de la costa pacifica de Costa Rica. Tesis de Licenciatura no publicada. Universidad Nacional, Costa Rica.

Víquez, R., Bolaños, R., Rodríguez, J., Palacios, J. A. \& Protti, M. (1992). Utilización de variables morfométricas para explicar el comportamiento del peso fresco y eviscerado en tres especies de peces del género Opisthonema (Pisces: Clupeidae). Uniciencia, 9, 57-69.

Víquez, R., Rodríguez, J., Palacios, J. A. \& Protti, M. (1990). Diferencias morfométricas por sexo de tres especies del género Opisthonema (Pisces: Clupeidae) en la costa del Pacífico, Costa Rica. Uniciencia, 7, 27-32. 